\title{
Downregulation of $\mathrm{HI} 9$ decreases the radioresistance in esophageal squamous cell carcinoma cells
}

This article was published in the following Dove Press journal: OncoTargets and Therapy

\begin{abstract}
Wenguang Luo, ${ }^{1,2}$ Wei Liu, ${ }^{2}$ Jie Yao, ${ }^{3}$ Wenjing Zhu, ${ }^{4}$ Hongyan Zhang, ${ }^{2}$ Qi Sheng, ${ }^{2}$ Lin Wang, ${ }^{2}$ Lei Lv, ${ }^{5}$ Liting Qian ${ }^{6}$

'School of Medicine, Shandong University, Jinan, Shandong 250100, People's Republic of China; ${ }^{2}$ Department of Radiation Oncology, Anhui Provincial Hospital, The First Affiliated Hospital of University of Science and Technology of China, Hefei, Anhui 23003I, People's Republic of China; ${ }^{3}$ Department of Oncology, The 161 Hospital of PLA, Wuhan, Hubei Province 4300I0, People's Republic of China; ${ }^{4}$ Department of Geriatric Medicine, Anhui Provincial Hospital, The First Affiliated Hospital of University of Science and Technology of China, Hefei, Anhui 23003I, People's Republic of China; ${ }^{5}$ Cancer Epigenetics Program, Anhui Cancer Hospital, The First Affiliated Hospital of University of Science and Technology of China, Hefei, Anhui 23003I, People's Republic of China; ${ }^{6}$ Department of Radiation Oncology, Anhui Provincial Cancer Hospital, Anhui Provincial Hospital, The First Affiliated Hospital of University of Science and Technology of China, Hefei, Anhui 23003I, People's Republic of China
\end{abstract}

Correspondence: Liting Qian Department of Radiation Oncology, Anhui Provincial Cancer Hospital, Anhui Provincial Hospital, The First Affiliated Hospital of University of Science and Technology of China, 17 Lujiang Road, Hefei, Anhui 23003I, People's Republic of China

Tel +86I 396 67I 6720

Email money2004@sina.com
Background: Radiotherapy is one of the most common treatments for esophageal squamous cell carcinoma (ESCC). Radioresistance is a major obstacle that limits the efficacy of radiotherapy. $\mathrm{H} 19$ has been considered as a factor affecting radioresistance, whereas the specific mechanism of H19 in ESCC radioresistance remains to be further elucidated.

Purpose: The objective of this study was to identify the relationship between H19 and radioresistance. The findings are expected to provide new insights into the treatment of radioresistant ESCC.

Methods: The expression levels of H19 in ESCC was analyzed using the online database starBase. The Oncomine database was used to further verify the association between H19 expression and patient age, gender, and tumor stage. The overall survival rates of ESCC patients were analyzed using the KM plotter database. Clonogenic survival was conducted to identify the value of survival fraction. The optical density values were obtained via MTS assays. Cells migration and stemness were observed through Transwell and sphere formation assays. The expression levels of H19, miR-22-3p and WNT1 were analyzed using qPCR.

Results: In our study, we firstly screened the H19 according to the online database starBase, and then the Oncomine database and KM plotter database showed that H19 expression was significantly upregulated in the ESCC tissues and associated with poor prognosis. Secondly, an ESCC radioresistant cell line, KYSE150R was established. Clonogenic survival showed that radiation decreased the value of survival fraction. MTS assays suggested that optical density values in KYSE150R cells were significantly higher than that in KYSE150 cells. Transwell and sphere formation assays showed radiation enhanced cell migration and stemness in ESCC cells. In addition, qPCR showed that H19 was upregulated in KYSE150R cells, and survival fraction assays showed that knockdown of H19 decreased the survival fraction values. MTS assays, migration and invasion assays suggested that H19 inhibited cells proliferation, migration and stemness in radioresistant KYSE150 cells. Moreover, qPCR assay showed that miR-22-3p expression levels was downregulated, but WNT1 was upregulated in KYSE150R cells as well as protein levels. Luciferase activity assay further showed that miR-22-3p inhibits the WNT1 expression.

Conclusion: Our results demonstrate that H19 knockdown downregulates the WNT1 via upregulating miR-22-3p expression, which leads to the inhibition of cells proliferation, migration and stemness in the radioresistant ESCC cells.

Keywords: ESCC, radioresistance, KYSE150R, H19, miR-22-3p, WNT1

\section{Introduction}

Esophageal cancer (EC) is a malignant cancer that occurs in the esophageal epithelial tissue, and is common tumor and one of leading causes of mortality worldwide. ${ }^{1}$ Based on the differences in histology, EC is divided into two main 
subtypes: Esophageal adenocarcinoma (EA) and Esophageal squamous cell carcinoma (ESCC). ${ }^{2}$ Generally, ESCC accounts for $90 \%$ of EC and is the predominant histological type versus EC in China. ${ }^{3}$ For ESCC treatments, radiation therapy is one of the commonly used methods, which can be used alone or in combination with chemotherapy and surgery. ${ }^{4}$ Despite great advances in radiotherapy technology, tumor recurrence and metastasis caused by radioresistant cancer cells lead to the increased mortality and the poor overall 5-year survival rate in ESCC patients. ${ }^{5}$ Currently, few clinical approaches are able to predict the effects of radiation therapy in patients with cancer or its effect on the radiosensitivity of cancer cells. Thus, it is significant to investigate mechanisms that promote radiation resistance in ESCC. Meanwhile, identifying more biomarkers that involved in ESCC are expected to improve the efficacy of radiotherapy.

Cancer stem cells (CSCs), accounting for a small part of the tumor mass, are a small proportion of tumor cells with the capabilities of self-renewal and differentiation. ${ }^{6}$ CSCs have been identified as the main contributors for the recurrence and metastasis in tumors. $^{7-10}$ In addition, the correlation between IncRNAs and CSCs-related transcriptional factors expression (including OCT4, SOX2 and NANOG) has been unveiled. ${ }^{11,12}$ More importantly, lncRNAs are associated with stemness, such as, B4GALT1-AS1 promotes colon cancer cell stemness. ${ }^{13}$ NEAT1 enhances the radioresistance of cervical cancer. ${ }^{14}$ FAM83H-AS1 contributes to the radioresistance, proliferation, and metastasis in ovarian cancer. ${ }^{15}$

Using bioinformatic analysis, it showed the aberrant expression of $\mathrm{H} 19$ in ESCC tissues and the correlation between $\mathrm{H} 19$ and poor prognosis. H19, an imprinted lncRNA, is one of the first identified lncRNAs. ${ }^{16}$ Accumulating evidence reveals that the dysregulated $\mathrm{H} 19$ acts as the tumorigenic and anti-tumorigenic roles in many tumors, such as bladder cancer, ${ }^{17}$ breast cancer. ${ }^{18}$ Huang et al have reported that $\mathrm{H} 19$ are associated with cell invasion and metastasis in EC. ${ }^{19}$ It has been found that H19 plays some certain role in the alteration of radio/chemoresistance in hepatocellular carcinoma cells. ${ }^{6}$ H19 also is associated with poor prognosis in breast cancer patients and promotes cancer stemness. ${ }^{20}$ In addition, H19 interacted with miR-130a-3p and miR$17-5 p$ to modify radioresistance. ${ }^{21}$
Many reports have revealed that the implication of Wnt signaling in various cancers. ${ }^{22,23}$ Meanwhile, researchers also revealed its roles in radioresistance, such as LIG4 mediates Wnt signalling-induced radioresistance, ${ }^{24}$ microRNA-324-3p regulates nasopharyngeal carcinoma radioresistance by directly targeting WNT2B ${ }^{25}$ The inhibitory role of miR-301a in cell migration and the facilitating role of miR-301a in radiosensitivity have been found in radioresistant-ESCC cells. ${ }^{26} \mathrm{Wnt}$ proteins are involved in the differentiation of stem cells via the $\mathrm{Wnt} / \beta$-catenin signaling pathway ${ }^{27}$ or affect stem cell fate. ${ }^{28}$ The activation of Wnt/ $\beta$-catenin signaling pathways is required for human embryonic stem cells. ${ }^{29}$ Moreover, Wnt pathway participated in inhibition of mouse embryonic stem cell differentiation. ${ }^{30}$ However, little is known about the relationship between $\mathrm{H} 19$ and Wnt signaling in in radioresistant ESCC cells.

In this current study, we aimed to elucidate the role of H19 in radioresistant ESCC cells. We firstly found that H19 is upregulated in the ESCC cells and associated with poor prognosis. Then, we established radio-resistant KYSE150 cells line, KYSE150R. The results showed that radiation enhanced cells migration and stemness in ESCC cells. Furthermore, we observed that knockdown of H19 inhibits cells proliferation, migration, and stemness in radioresistant KYSE150 cells. In addition, knockdown of H19 downregulates WNT1 expression. Overexpression of miR-22-3p inhibits WNT1 expression. Our findings suggested that $\mathrm{H} 19$ promotes cells proliferation, migration, and stemness in the ESCC radioresistant cells.

\section{Materials and methods}

\section{Bioinformatic analysis}

The expression levels of H19 in ESCC was analyzed using the online database starBase (http://starbase.sysu.edu.cn/). The publicly available Oncomine database (http://www. oncomine.com) was utilized to further verify the association between $\mathrm{H} 19$ expression and patient age, gender, and tumor stage. The overall survival rates of ESCC patients were analyzed using the KM plotter database (http:// kmplot.com/). Additionally, the starBase database was also used to assess the combination between H19, miR22-3p and WNT1 in ESCC cells. The binding sites of miR-22-3p in WNT1 were predicted using the bioinformatics analysis tool TargetScan. 


\section{Establishment of a radioresistant ESCC} cell line and cell culture

ESCC cell line KYSE150 was purchased from the Cell Bank of Shanghai Institutes for Biological Sciences, Chinese Academy of Sciences (Shanghai, China). KYSE150 cells were cultured in RPMI-1640 medium (Gibco, Carlsbad, CA, USA) supplemented with $10 \%$ fetal bovine serum (FBS, Hyclone, Logan, USA). After reaching 50 to $60 \%$ confluence, KYSE150 cells were exposed to 2 Gy X-ray irradiation (2.5 Gy/min) using a Varian-6/100 Linear Accelerator (Varian Medical Systems, Inc., Palo Alto, CA, USA). Next, the medium was changed into the RPMI-1640 culture medium (Gibco, Carlsbad, CA, USA) containing 10\% fetal bovine serum (FBS, Hyclone, Logan, USA). The culture medium was changed every two days. $15 \%$ FBS was employed when numerous dead cells were observed. Cells were further maintained in a humidified incubator with $5 \% \mathrm{CO}_{2}(\mathrm{v} / \mathrm{v})$. After reaching $70 \%$ to $80 \%$ confluence, cells were passaged. Repeat the above process until the total radiation reached 60 Gy. The obtained cells were designated KYSE150R cells, and further cultured for $\geq 2$ weeks before the subsequent experiments.

\section{HI9 knockdown assays}

Specific small interfering RNA (siRNAs) for H19 and negative control siRNA (NC-si) were obtained from RiboBio (Guangzhou, China). KYSE150R cells were planted into 12well plates $\left(1 \times 10^{6} /\right.$ well) and incubated for $12 \mathrm{~h}$. H19-si or NCsi was transfected into cells using X-treme GENE transfection reagent (Roche, Basel, Switzerland) according to the manufacturer's instructions. After $48 \mathrm{~h}$ transfection, total RNA was isolated and used for analysis of the mRNA and protein expression. The sequences of H19-si in this study were as follows, siRNA GACACCAUCGGAACAGCAG and NC-si 5'-UUCUCCGAACGUGUCACGU-3' (sense).

\section{Clonogenic survival assays}

KYSE150 and KYSE150R cells transfected with H19-si or untransfected with H19-si were plated into 6-well plates $\left(\left(2 \times 10^{6} /\right.\right.$ well). Cells were incubated at $37{ }^{\circ} \mathrm{C}$ in a humidified incubator with $5 \% \mathrm{CO}_{2}$. After incubation for $24 \mathrm{~h}$, cells were irradiated with $0,2,4,6$, or 8 Gy using X-ray irradiation. After incubation for $48 \mathrm{~h}$, cells were washed twice with PBS, fixed in $70 \%$ methanol, and stained with $0.1 \%$ crystal violet. Colonies containing more than 50 cells were counted. The survival fraction of each dose was determined using the equation survival fraction $=$ colonies counted $/($ cells seeded $\times \mathrm{PE}) \times 100 \%$.

\section{Cell proliferation assays}

To determine the proliferative ability of cells, the MTS assays were performed. Cells were seeded into 96-well plates $\left(5 \times 10^{3} /\right.$ well) containing $100 \mu \mathrm{L}$ of $10 \% \mathrm{FBS} /$ medium and incubated at $37{ }^{\circ} \mathrm{C}$ with $5 \% \mathrm{CO}_{2}$. After incubation for 1 to 7 days, the MTS assays were conducted according to the manufacturer's instructions. The cell viability was determined by measuring the optical density (OD) at $490 \mathrm{~nm}$ wavelength using a spectrophotometer FP-6500 (JASCO Corp.).

\section{Transwell assays}

Transwell assays were performed in $6.5 \mathrm{~mm}$ Transwell chambers with $8 \mu \mathrm{m}$ pores (Corning Costar, Corning, NY, USA) to evaluate the migration ability of cells according to the manufacturer's instructions. The upper chamber was loaded with cells in serum-free medium. The lower chamber was filled with medium containing $10 \%$ FBS. After incubation for $24 \mathrm{~h}$, cells that had invaded through the membrane were fixed with methanol and stained with $0.1 \%$ crystal violet (SigmaAldrich, St. Louis, MO, USA). The number of cells in the lower chamber was counted using an inverted microscope ( $\times 100$ magnification) .

\section{qPCR analysis}

Total RNA was extracted from cells with Trizol reagent (Invitrogen, Carlsbad, CA, USA) according to the manufacturer's instructions. cDNA synthesis was performed using the high capacity cDNA Reverse Transcription Kit (Applied Biosystems, Foster City, CA). GAPDH and U6 were used as the internal control. All qPCR assays were performed with a CFX Connect ${ }^{\mathrm{TM}}$ Real-Time PCR Detection System (BIORAD Laboratories, Inc., California, USA), and the fold changes were calculated by the relative quantification $\left(2^{-\Delta \Delta \mathrm{Ct}}\right)$ method. The primers were shown in Table 1.

\section{Sphere-forming assays}

Cells were digested using $0.25 \%$ trypsin (Sigma); washed twice using calcium/magnesium-free phosphate-buffered saline (PBS); suspended in serum-free DMEM-F12 medium supplemented with $1 \%$ penicillin-streptomycin solution, $20 \mathrm{ng} / \mathrm{mL}$ epidermal growth factor, $10 \mathrm{ng} / \mathrm{mL}$ basic fibroblast growth factor, and $2 \% \mathrm{~B} 27$; and then seeded in a lowattachment T25 flask (Corning). Cells were collected after 5 days, and then digested using Accutase enzyme and seeded into 96-well plates. Cells were cultivated for 7-14 days, and the spheres were then assessed using a microscope. 
Table I The primers used for qPCR were listed

\begin{tabular}{|l|l|l|}
\hline Gene & \multicolumn{3}{|l|}{ Primer sequence (5'-3') } \\
\hline HI9 & $\begin{array}{l}\text { Forward } \\
\text { Reverse }\end{array}$ & $\begin{array}{l}\text { TTACTGCTGCGTTTTATGTTGGG } \\
\text { GCTGGCCGATGTGATGACTA }\end{array}$ \\
\hline miR-22-3p & $\begin{array}{l}\text { Forward } \\
\text { Reverse }\end{array}$ & $\begin{array}{l}\text { GGGAAGCTGCCAGTTGAAG } \\
\text { GTGCGTGTCGTGGAGTCG }\end{array}$ \\
\hline WNTI & $\begin{array}{l}\text { Forward } \\
\text { Reverse }\end{array}$ & $\begin{array}{l}\text { TTCAGACACGAGAGATGGAACT } \\
\text { CCAGCCTTCACTTGCTGAG }\end{array}$ \\
\hline OCT4 & $\begin{array}{l}\text { Forward } \\
\text { Reverse }\end{array}$ & $\begin{array}{l}\text { GTGTTCAGCCAAAAGACCATCT } \\
\text { GGCCTGCATGAGGGTTTCT }\end{array}$ \\
\hline SOX2 & $\begin{array}{l}\text { Forward } \\
\text { Reverse }\end{array}$ & $\begin{array}{l}\text { CTCGTGCAGTTCTACTCGTCG } \\
\text { AGCTCTCGGTCAGGTCCTTT }\end{array}$ \\
\hline NANOG & $\begin{array}{l}\text { Forward } \\
\text { Reverse }\end{array}$ & $\begin{array}{l}\text { TCCCGAGAAAAGATTAGTCAGCA } \\
\text { AGTGGGGCACCTGTTTAACTT }\end{array}$ \\
\hline GAPDH & $\begin{array}{l}\text { Forward } \\
\text { Reverse }\end{array}$ & $\begin{array}{l}\text { GGGAGCCAAAAGGGTCAT } \\
\text { GAGTCCTTCCACGATACCAA }\end{array}$ \\
\hline
\end{tabular}

\section{Western bolt analysis}

Cells were grown in 6-well plates $\left(1 \times 10^{6}\right)$ and transfected with the indicated plasmids and siRNA using Lipofectamine 2000 (Thermo Fisher Scientific). Cells were washed with precooling phosphate-buffered saline (PBS), and cells were collected and then lysed for 20 mins in cold lysis buffer (Beyotime Institute of Biotechnology). Extracts were clarified by centrifugation $(12,000 \mathrm{rpm} / \mathrm{min})$ for $30 \mathrm{mins}$ at $4{ }^{\circ} \mathrm{C}$. Protein samples were resolved by electrophoresing on $10 \%$ polyacrylamide gel electrophoresis (SDS-PAGE) and then transferred to polyvinylidene fluoride (PVDF). Membranes were blocked in Tris-buffered saline (TBS) containing 5\% nonfat dry milk and $0.05 \%$ Tween- 20 for 20 mins with shaking and then incubated with primary antibody following the manufacturer's instructions for $2 \mathrm{hrs}$ at room temperature. Samples were then washed with TBST (TBS-0.1\% Tween-20) and incubated for $1 \mathrm{hr}$ with horseradish peroxidase-conjugated secondary antibody (1:10,000 dilution). Protein bands were visualized using enhanced chemiluminescent substrate according to the manufacturer's protocol (Millipore, Billerica, MA, USA). The primary antibodies were WNT1 and $\beta$-actin from ABclonal Biotechnology (Wuhan, P.R. China).

\section{Luciferase activity assays}

The DNA sequences of 3'-UTR-wide type of WNT1 (WNT1WT) and the corresponding mutant vector 3'-UTR-mutant type of WNT1 (WNT1-MUT) were separately synthesized and cloned into the pGL-3 basic vector (Promega, Madison, WI, USA). Cells were planted into 24-well plates, and then cells were co-transfected with WNT1-WT or WNT1-MUT plasmids, miR-22-3p mimic or miR-NC (RiboBio, Guangzhou, China), and pRL-TK plasmids (Promega, Madison, WI, USA). Cells were collected and then luciferase activity were measured using the Dual-Luciferase Reportor Assay System (Promega, Madison, WI, USA).

\section{Statistical analysis}

Each experiment was repeated in triplicate. Unless other noted, all data were presented as the mean \pm SD. The SPSS version 17.0 (SPSS, Chicago, IL, USA) was used to perform all statistical analyses. Unless otherwise mentioned, one-way ANOVA was used for analyzing the difference in multiply group ( $>2)$. Two-way ANOVA was used for analyzing the differences in luciferase activity assay. $P<0.05$ was considered statistically significant.

\section{Results}

\section{$\mathrm{HI}$ is upregulated in the ESCC and associated with poor prognosis}

To seek more the ESCC related lncRNAs, the online database starBase was firstly used. We found that H19 was upregulated (Figure 1A). Based on the Oncomine database, we found that $\mathrm{H} 19$ expression had no effects on gender, clinical stage IV, and age (Figure 1B-D). However, data retrieved from the KM plotter database showed that H19 expression was associated with a poor prognosis in ESCC patients (Figure 1E, $P=0.0052$ ). Collectively, these results suggest that $\mathrm{H} 19$ is upregulated in the ESCC and associated with poor prognosis.

\section{Radiation enhanced cells migration and stemness}

To explore the effect of radiation on ESCC cells, we established the resistant cells in KYSE150 and KYSE510 (failed). Therefore, we conducted clonogenic survival and cell viability assays to verify the resistant KYSE150 cells, KYSE150R. The results showed that radiation decreased the value of survival fraction with increasing of radiation doses (Figure 2A, $P<0.05$ ). Subsequently, MTS assays were conducted to analyze cells viability. We observed that the OD values in KYSE150R cells were significantly higher than that in KYSE150 cells. (Figure 2B, $P<0.05$ ). Taken together, 
A

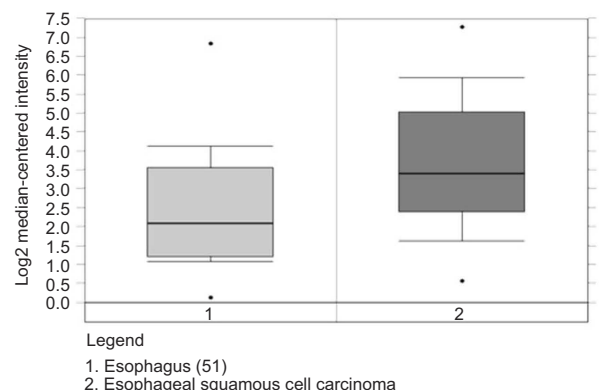

D

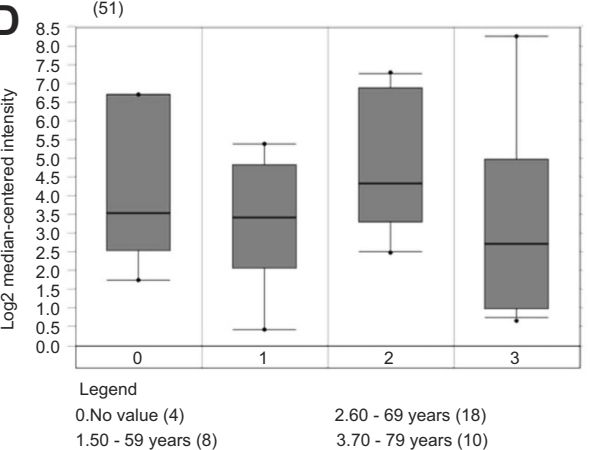

B



E



C

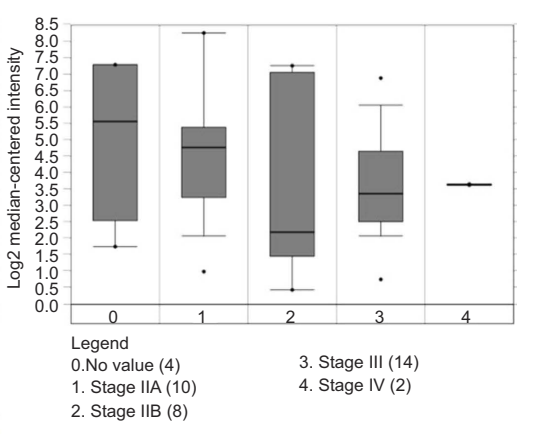

Figure I HI9 is upregulated in the ESCC and associated with poor prognosis. (A) Online database starBase suggested that HI9 was upregulated. (B-D) Oncomine database showed that HI9 expression had no effects on gender, clinical stage IV, and age. (E) The KM plotter database showed that HI9 expression was associated with a poor prognosis in ESCC patients.

A

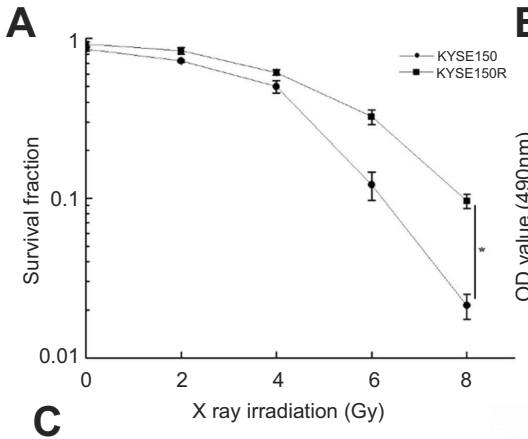

C

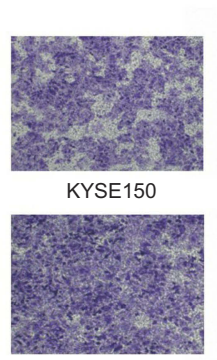

KYSE150R

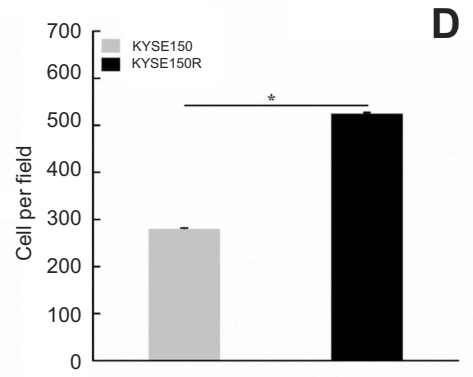

D


Figure 2 Radiation enhanced cells migration and stemness. (A) Radiation decreased the value of survival fraction with increasing of radiation doses. (B) MTS assays showed that the OD values in KYSEI50R cells were significantly higher than that in KYSEI50 cells. (C) Transwell assays indicated that cells migration ability was higher in KYSEI50R. (D) Sphere formation assays showed that sphere formation ability was enhanced in KYSEI50R cells. (E) qPCR assays implied that stemness-associated genes (OCT4, SOX2 and NANOG) were significantly higher in KYSEI50R cells. $* P<0.05$. 
we successfully constructed the KYSE150R cells. Therefore, we further performed related functional experiments. Transwell assays were performed. The results showed that cells migration ability was higher in KYSE150R (Figure 2C, $P<0.05$ ). Then, sphere formation assays were conducted. The results suggested that the sphere formation ability was enhanced in KYSE150R cells (Figure 2D). Furthermore, qPCR assays were performed in KYSE150R and KYSE150 cells. We found that the stemness-associated genes (OCT4, SOX2 and NANOG) were significantly higher in KYSE150R cells. (Figure $2 \mathrm{E}, P<0.05$ ). Conjointly, these results indicate that radiation enhanced cells migration and stemness in ESCC cells.

\section{Knockdown of HI9 inhibits cells proliferation, migration, and stemness in radioresistant $\mathrm{KYSEI} 50$ cells}

To explore the role of H19 in ESCC radioresistant cells, qPCR assays were performed. We found that H19 expression was significantly upregulated in KYSE150R cells. (Figure 3A, $P<0.05$ ). Then, survival fraction assays were conducted. H19-si was transfected into KYSE150R.
We found that knockdown of H19 decreased the survival fraction values with the increasing of radiation doses (Figure 3B, $P<0.05$ ). MTS and transwell assays were conducted. The results suggested that knockdown of H19 inhibited cells proliferation and migration (Figure 3C, D, $P$ $<0.05)$. In addition, the sphere formation assays were conducted. The results showed that knockdown of H19 markedly decreased the sphere formation ability of KYSE150R cells (Figure 3E, $P<0.05$ ). Moreover, the, the levels of the stemness-associated genes were identified using qPCR. We found that knockdown of H19 restrained OCT4, SOX2, and NANOG expression levels (Figure 3F, $P<0.05)$. Taken together, these results revealed that knockdown of H19 inhibits cells proliferation, migration, and stemness in radioresistant KYSE150 cells.

\section{Knockdown of HI9 downregulates WNTI expression}

Based on the online starBase database, data showed that H19 and WNT 3'UTR contain the binding sites of miR-223p (Figure 4A). Knockdown experiment was conducted. H19-si was transfected into KYSE150R cells and the results showed that knockdown of H19 increased the levels of
A

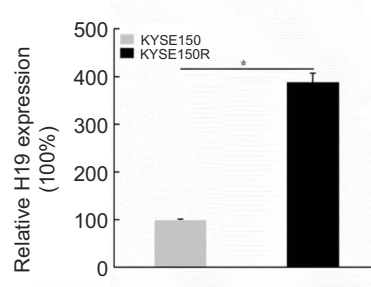

B



D

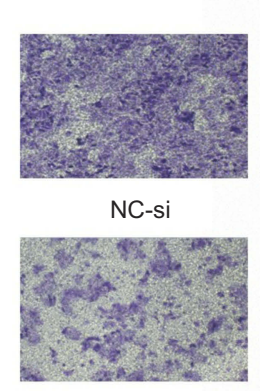

H19-si

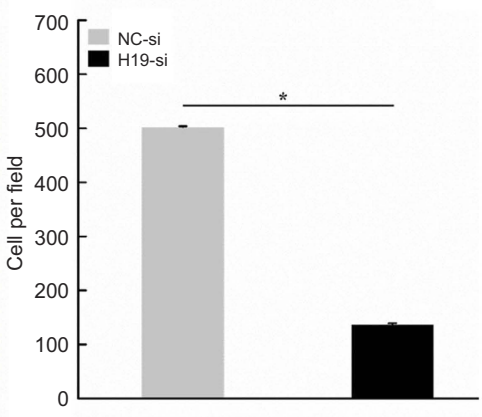

E

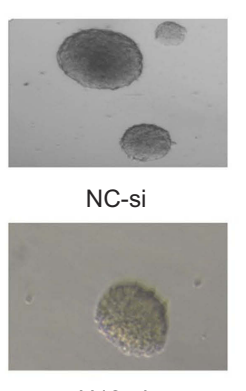

H19-si
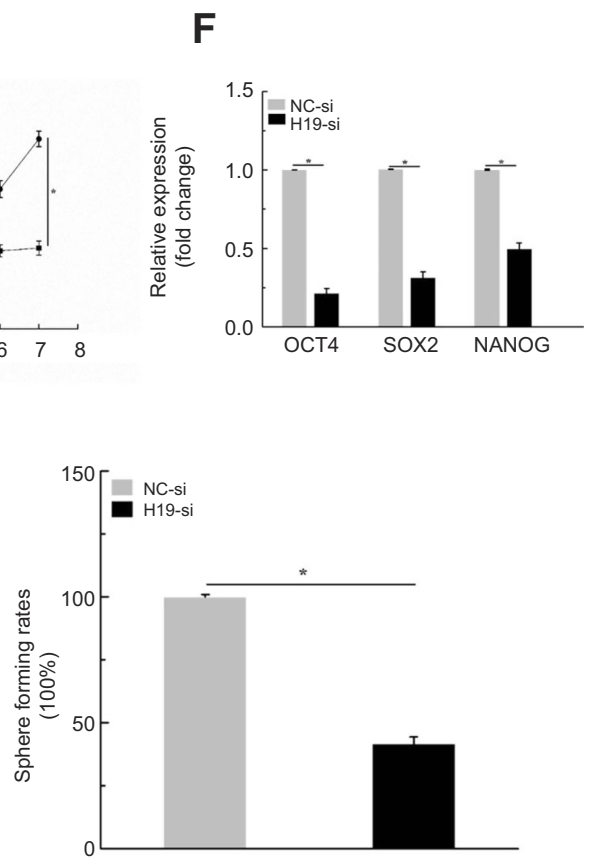

Figure 3 Knockdown of HI9 inhibits cells proliferation, migration, and stemness in radioresistant KYSEI50 cells. (A) qPCR assays showed that HI9 expression was significantly upregulated in KYSEI 50R cells. (B) knockdown experiment suggested that downregulation of HI9 decreased the survival fraction values with the increasing of radiation doses. (C and D) MTS and transwell assays indicated that knockdown of HI9 inhibited cells proliferation and migration ability. (E) Sphere formation assays showed that knockdown of HI9 markedly decreased the sphere formation ability of KYSEI50R cells. (F) qPCR assays implied that knockdown of HI9 restrained OCT4, SOX2, and NANOG expression levels. *P<0.05. 
A

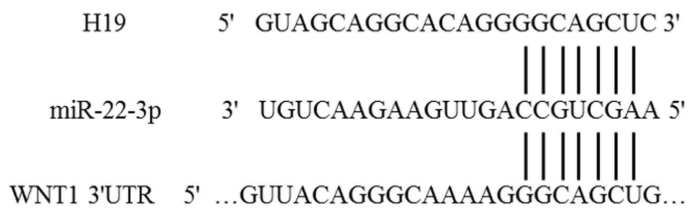

C

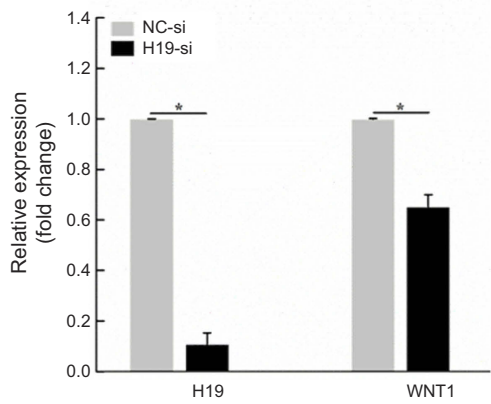

B

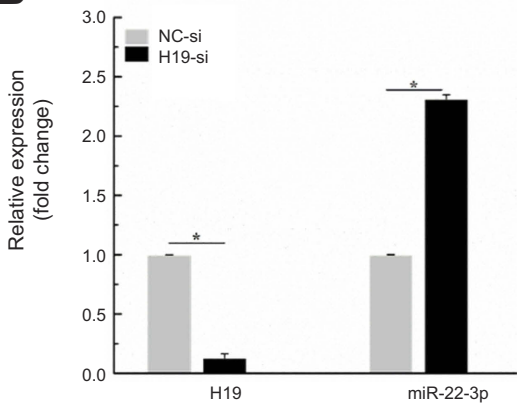

D

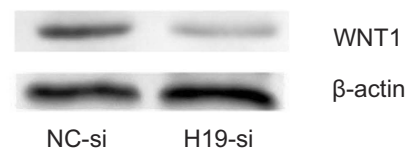

Figure 4 Knockdown of HI9 downregulates WNTI expression. (A) Online starBase database showed that HI9 and WNT 3'UTR contain the binding sites of miR-22-3p. (B) Knockdown experiment suggested that downregulation of HI9 increased the levels of miR-22-3p expression (C, D) Knockdown of HI 9 significantly decreased WNTI expression at both the mRNA and protein levels in KYSEI50R cells. $* P<0.05$.
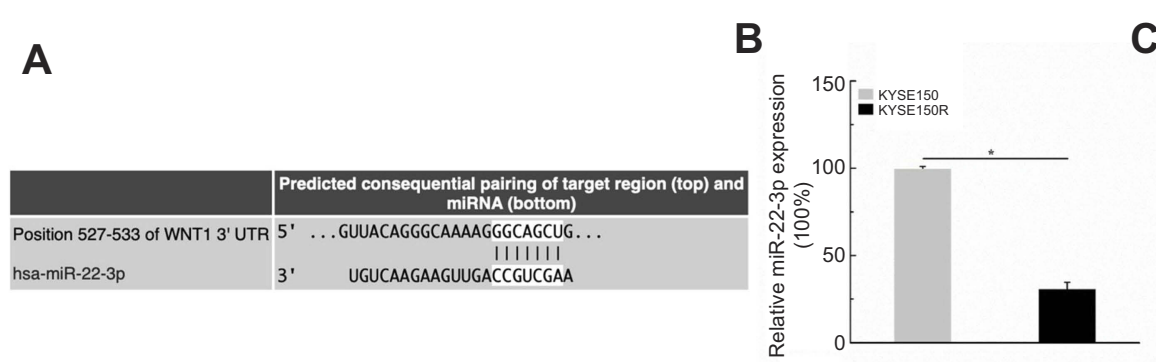

C

D

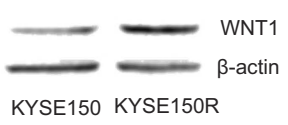

E

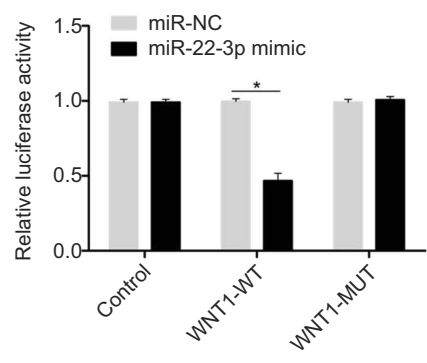

$\mathbf{F}$

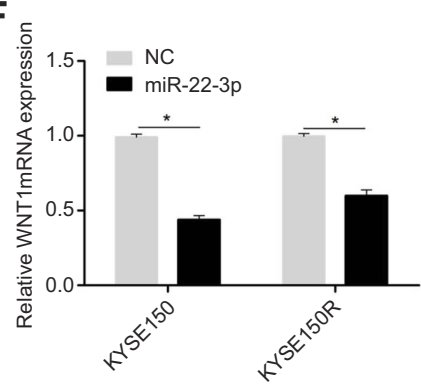

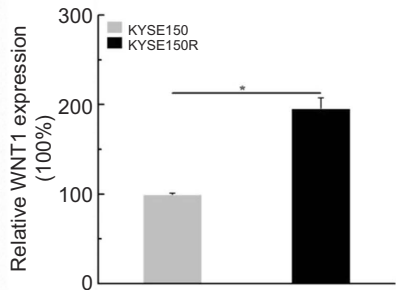

G

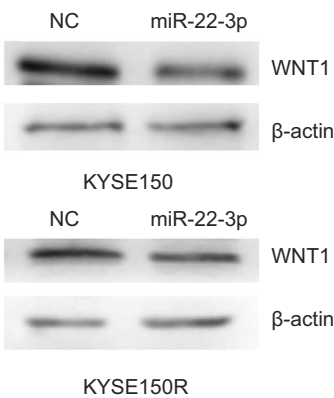

Figure 5 Overexpression of miR-22-3p inhibits WNTI expression. (A) TargetScan showed that WNTI 3'UTR contained the binding sites of miR-22-3p (B, C) qPCR assays suggested that miR-22-3p expression levels were downregulated, but WNTI upregulated in KYSEI50R cells. (D) Western blot assays showed WNTI protein levels were upregulated in KYSEI50R. (E) Luciferase activity assay showed a significant reduction after co-transfection of miR-22-3p mimic in WNTI-WT group, but no significant changes in the WNTI-MUT. (F, G) qPCR and Western blot assays showed overexpression miR-22-3p inhibits WNTI expression. $* P<0.05$.

miR-22-3p expression (Figure 4B, $P<0.05$ ). In addition, we further analyzed the association between H19 and WNT1.
The results showed that knockdown of H19 significantly decreased WNT1 expression at both the mRNA and protein 
levels in KYSE150R cells (Figure 4C, D). Taken together, knockdown of H19 upregulates miR-22-3p, while downregulates WNT1 expression.

\section{Overexpression of miR-22-3p inhibits WNTI expression}

To further explore the relationship of miR-22-3p and WNT1, the bioinformatics analysis tool TargetScan was used. The results showed that WNT1 3'UTR contained the binding sites of miR-22-3p (Figure 5A). Then, qPCR assays were conducted and the results showed that miR-22-3p expression levels were downregulated, but WNT1 upregulated in KYSE150R cells. (Figure 5B, C, $P<0.05$ ). Moreover, Western blot assays showed WNT1 protein levels were upregulated in KYSE150R. (Figure 5D). Luciferase activity assay showed a significant reduction after co-transfection of miR-22-3p mimic in WNT1-WT group, but not in the WNT1-MUT (Figure 5E, $P<0.05$ ). Furthermore, miR-22-3p mimic and NC were transfected into KYSE150 and KYSE150R cells, separately. qPCR and Western blot assays were performed to exam the WNT1 expression. The findings suggested that overexpression of miR-22-3p inhibits WNT1 expression (Figure 5F, G). Collectively, our results indicated that miR-22-3p targets WNT1 expression.

\section{Discussion}

Despite radiotherapy is a common treatment for ESCC, radioresistance always occurs and limits the use of radiotherapy. Resistance to radiation therapy leads to high tumor recurrence rates, cancer metastasis, and poor prognosis, and therefore clarifying the mechanism of radioresistance is essential to control tumor growth during radiation therapy. ${ }^{31}$ In this study, the upregulation of H19 in the ESCC tissues and the association between H19 and poor prognosis were obtained using the Oncomine database. Although the mechanisms of tumor radioresistance are complicated, studies with respect to the improvement of radiosensitization have achieved continuous progression. ${ }^{32}$

LncRNAs regulate the transcription of genes associated with DNA damage response, which is closely correlated with sensitivity to radiation therapy. The potential associations between IncRNAs and stemness have also been identified. For example, lncRNA HOTAIR regulates cell viability and radiosensitivity through inhibiting miR-218 in colorectal cancer. ${ }^{33}$ H19 is aberrantly expressed in various cancers and plays an pivotal role in enhancing stemness of cancer cells. ${ }^{34}$ CASC11 increases cancer cell stemness and predicts postoperative survival in small cell lung cancer. Moreover, previous evidence has revealed that CSCs play important roles in cancer therapy. ${ }^{10,35-40}$

In addition, the interactions between IncRNAs and miRNAs in tumor cells are implicated in tumor progression or regression. ${ }^{41} \mathrm{H} 19$ has been reported to target miR194-5p, which was essential for development of colorectal adenocarcinoma. ${ }^{42}$ Moreover, miRNAs have been widely identified involved in radiosensitivity. NRF2 as a new potential molecular target whose inhibition might represent a novel radiosensitizing in rhabdomyosarcoma. ${ }^{43}$ microRNA-153-3p enhances cell radiosensitivity by targeting BCL2 in human glioma. $^{44}$ microRNA-16-5p enhances radiosensitivity in prostate tumor cells. ${ }^{45}$ miR122 has been found as a tumor suppressor to influence the radioresistance in breast cancer. ${ }^{46}$ In addition,

One study showed that microRNA 301a targets WNT1 to suppress cell proliferation and migration and enhance radiosensitivity in esophageal cancer cells.

In this study, we firstly screened the H19 according to the online database starBase. Then, Oncomine database and $\mathrm{KM}$ plotter database were used and the data showed that $\mathrm{H} 19$ is upregulated in the ESCC and associated with poor prognosis. To identify the role of radiation in the resistant KYSE150 cells. The resistant KYSE150 cells, KYSE150R was firstly established. Clonogenic survival showed that radiation decreased the value of survival fraction. MTS assays suggested that OD values in KYSE150R cells were significantly higher than that in KYSE150 cells. Subsequently, we further performed related functional experiments. Transwell and sphere formation assays showed that cells migration and sphere formation ability were higher in KYSE150R, suggesting that radiation enhanced cells migration and stemness in ESCC cells. To figure out the role of H19 in ESCC radioresistant cells, qPCR firstly showed that H19 expression was significantly upregulated in KYSE150R cells. Furthermore, survival fraction assays showed that knockdown of H19 decreased the survival fraction values. A series of functional experiments indicated that H19 inhibited cells proliferation, migration and stemness in radioresistant KYSE150 cells. Moreover, starBase database suggested that $\mathrm{H} 19$ and WNT1 3'UTR contained the miR-22-3p binding sites. In KYSE150R cells, qPCR assays showed that miR-22-3p expression levels was downregulated, but WNT1 upregulated as well as protein levels after transfected with H19-si. Moreover, luciferase activity assay further showed that 
miR-22-3p inhibits the WNT1 expression. Our findings may implicate that knockdown of $\mathrm{H} 19$ downregulates the WNT1 via upregulating miR-22-3p expression, which could contribute to the inhibition of cells proliferation, migration and stemness in the radioresistant ESCC cells.

\section{In conclusion}

Our preliminary findings demonstrate that H19 promotes cells proliferation, migration, and stemness in the ESCC radioresistant cells. The more specified mechanisms remain to be elucidated and therefore additional researches with a large number of clinical samples are required for the application of targeted therapy for ESCC radioresistance. We will further investigate the potential role of H19 in ESCC radioresistance.

\section{Acknowledgment}

This work was supported by grants from The Natural Science Foundation of Anhui Province (NO. 1808085MH266).

\section{Disclosure}

The authors report no conflicts of interest in this work.

\section{References}

1. Domper Arnal MJ, Ferrandez Arenas A, Lanas Arbeloa A. Esophageal cancer: risk factors, screening and endoscopic treatment in Western and Eastern countries. World J Gastroenterol. 2015;21 (26):7933-7943. doi:10.3748/wjg.v21.i26.7933

2. Yang W, Ma J, Zhou W, et al. Molecular mechanisms and clinical implications of miRNAs in drug resistance of esophageal cancer Expert Rev Gastroenterol Hepatol. 2017;11(12):1. doi:10.1080/ 17474124.2017.1372189

3. Hinrichs CS, van Way CW. Esophageal cancer. Curr Surg. 2002;59 (1):12-17.

4. Baskar R, Itahana K. Radiation therapy and cancer control in developing countries: can we save more lives? Int J Med Sci. 2017;14 (1):13-17. doi:10.7150/ijms. 17288

5. Le Bras GF, Farooq MH, Falk GW, Andl CD. Esophageal cancer: the latest on chemoprevention and state of the art therapies. Pharmacol Res. 2016;113(Pt A):236-244. doi:10.1016/j.phrs.2016.08.021

6. Ma H, Yuan L, Li W, Xu K, Yang L. The LncRNA H19/miR-193a-3p axis modifies the radio-resistance and chemotherapeutic tolerance of hepatocellular carcinoma cells by targeting PSEN1. J Cell Biochem. 2018. doi: $10.1002 /$ jcb. 26883

7. Qiu H, Fang X, Luo Q, Ouyang G. Cancer stem cells: a potential target for cancer therapy. Cell Mol Life Sci. 2015;72(18):3411. doi:10.1007/s00018-015-1920-4

8. Sharifzad F, Ghavami S, Mardpour S, et al. Glioblastoma cancer stem cell biology: potential theranostic targets. Drug Resist Updat 2019;42:35-45. doi:10.1016/j.drup.2018.03.003

9. Toden S, Kunitoshi S, Cardenas J, et al. Cancer stem cell-associated miRNAs serve as prognostic biomarkers in colorectal cancer. $J C I$ Insight. 2019;4:6. doi:10.1172/jci.insight. 122686

10. Dzobo K, Senthebane DA, Rowe A, et al. Cancer stem cell hypothesis for therapeutic innovation in clinical oncology? taking the root out, not chopping the leaf. Omics. 2016;20(12):681-691. doi:10.1089/omi.2016.0152
11. Fu Z, Chen C, Zhou Q, et al. LncRNA HOTTIP modulates cancer stem cell properties in human pancreatic cancer by regulating HOXA9. Cancer Lett. 2017;410:68. doi:10.1016/j.canlet.2017.09.019

12. Pan Y, Li C, Chen J, et al. The emerging roles of long noncoding RNA ROR (lincRNA-ROR) and its possible mechanisms in human cancers. Cell Physiol Biochem. 2016;40(1-2):219-229. doi:10.1159/000452539

13. Zhang Y, Fang Z, Guo X, et al. IncRNA B4GALT1-AS1 promotes colon cancer cell stemness and migration by recruiting YAP to the nucleus and enhancing YAP transcriptional activity. J Cell Physiol. 2019.

14. Han D, Wang J, Cheng G. LncRNA NEAT1 enhances the radio-resistance of cervical cancer via miR-193b-3p/CCND1 axis. Oncotarget. 2018;9(2):2395-2409. doi:10.18632/oncotarget.23416

15. Dou Q, Xu Y, Zhu Y, Hu Y, Yan Y, Yan H. LncRNA FAM83H-AS1 contributes to the radioresistance, proliferation, and metastasis in ovarian cancer through stabilizing HuR protein. Eur J Pharmacol. 2019;852:134-141. doi:10.1016/j.ejphar.2019.03.002

16. Cai X, Cullen BR. The imprinted H19 noncoding RNA is a primary microRNA precursor. $R N A$. 2007;13(3):313-316. doi:10.1261/rna.351707

17. Lv M, Zhong Z, Huang M, Qiang T, Rong J, Chen J. IncRNA H19 regulates epithelial-mesenchymal transition and metastasis of bladder cancer by miR-29b-3p as competing endogenous RNA. Biochim Biophys Acta. 2017;1864:10.

18. Zhou ZR, Yang ZZ, Yu XL, Guo XM. Highlights on molecular targets for radiosensitization of breast cancer cells: current research status and prospects. Cancer Med. 2018;7(7):3110-3117. doi:10.1002/cam4.1588

19. Huang C, Cao L, Qiu L, et al. Upregulation of H19 promotes invasion and induces epithelial-to-mesenchymal transition in esophageal cancer. Oncol Lett. 2015;10(1):291. doi:10.3892/ol.2015.3207

20. Shima H, Kida K, Adachi S, Yamada A. Lnc RNA H19 is associated with poor prognosis in breast cancer patients and promotes cancer stemness. Breast Cancer Res Treat. 2018;170(3):507-516. doi:10.1007/s10549-018-4793-z

21. Aalijahan H, Ghorbian S. Long non-coding RNAs and cervical cancer. Exp Mol Pathol. 2019;106:7-16. doi:10.1016/j.yexmp.2018.11.010

22. Bengoa-Vergniory N, Gorrono-Etxebarria I, Lopez-Sanchez I, Marra M, Di Chiaro P, Kypta R. Identification of noncanonical Wnt receptors required for wnt-3a-induced early differentiation of human neural stem cells. Mol Neurobiol. 2017;54(8):6213-6224. doi:10.1007/s12035-016-0151-5

23. Bengoa-Vergniory N, Kypta RM. Canonical and noncanonical Wnt signaling in neural stem/progenitor cells. Cell Mol Life Sci. 2015;72 (21):4157-4172. doi:10.1007/s00018-015-2028-6

24. Jun S, Jung YS, Han NS, et al. LIG4 mediates Wnt signalling-induced radioresistance. Nat Commun. 2016;7:10994. doi:10.1038/ncomms 10994

25. Li G, Liu Y, Su Z, et al. MicroRNA-324-3p regulates nasopharyngeal carcinoma radioresistance by directly targeting WNT2B. Eur $J$ Cancer. 2013;49(11):2596-2607. doi:10.1016/j.ejca.2013.03.001

26. Su H, Wu Y, Fang Y, et al. MicroRNA-301a targets WNT1 to suppress cell proliferation and migration and enhance radiosensitivity in esophageal cancer cells. Oncol Rep. 2019;41(1):599-607. doi:10.3892/or.2018.6799

27. Foulquier S, Daskalopoulos EP, Lluri G, Hermans KCM, Deb A, Blankesteijn WM. WNT signaling in cardiac and vascular disease. Pharmacol Rev. 2018;70(1):68-141. doi:10.1124/pr.117.013896

28. Van Camp JK, Beckers S, Zegers D, Van Hul W. Wnt signaling and the control of human stem cell fate. Stem Cell Rev. 2014;10 (2):207-229. doi:10.1007/s12015-013-9486-8

29. Dzobo K, Vogelsang M, Parker MI. Wnt/beta-catenin and MEK-ERK signaling are required for fibroblast-derived extracellular matrix-mediated endoderm differentiation of embryonic stem cells. Stem Cell Rev. 2015;11(5):761-773. doi:10.1007/s12015-015-9598-4

30. Wang Q, Song JW, Liu Y, Zhao XX. Involvement of Wnt pathway in ethanol-induced inhibition of mouse embryonic stem cell differentiation. Alcohol. 2017;58:13-18. doi:10.1016/j.alcohol. 2016.11.006 
31. Chi HC, Tsai CY, Tsai MM, Yeh CT, Lin KH. Roles of long noncoding rnas in recurrence and metastasis of radiotherapy-resistant cancer stem cells. Int J Mol Sci. 2017;18(9):1903. doi:10.3390/ijms18091903

32. Dongping W, Qiang Z, Schreiber JS, et al. Targeting mcl-1 for radiosensitization of pancreatic cancers. Transl Oncol. 2015;8 (1):47-54. doi:10.1016/j.tranon.2014.12.004

33. Li P, Zhang X, Wang L, et al. IncRNA HOTAIR contributes to 5FU resistance through suppressing miR-218 and activating NF- $\mathrm{kB} / \mathrm{TS}$ signaling in colorectal cancer. Mol Ther Nucleic Acids. 2017;8:356-369. doi:10.1016/j.omtn.2017.07.007

34. Gallula J, Schneider T, Barkali M. Oncofetal H19 RNA promotes tumor metastasis. Biochim Biophys Acta Mol Cell Res. 2014;1843 (7):1414-1426. doi:10.1016/j.bbamcr.2014.03.023

35. Eun K, Ham SW, Kim H. Cancer stem cell heterogeneity: origin and new perspectives on CSC targeting. BMB Rep. 2017;50(3):117-125.

36. Peiris-Pagès M, Martinez-Outschoorn UE, Pestell RG, Sotgia F, Lisanti MP. Cancer stem cell metabolism. Breast Cancer Res. 2016;18(1):55. doi:10.1186/s13058-016-0712-6

37. Shaheenah D, Laura A, Massimo C. Cancer stem cells: implications for cancer therapy. Oncology. 2014;28(12):1101.

38. Batlle E, Clevers H. Cancer stem cells revisited. Nat Med. 2017;23 (10):1124-1134. doi:10.1038/nm.4409

39. Vlashi E, Pajonk F. Cancer stem cells, cancer cell plasticity and radiation therapy. Semin Cancer Biol. 2015;31:28-35. doi:10.1016/j. semcancer.2014.07.001
40. Wang T, Shigdar S, Gantier MP, et al. Cancer stem cell targeted therapy: progress amid controversies. Oncotarget. 2015;6 (42):44191-44206. doi:10.18632/oncotarget.6176

41. Peng W, Shangwei N, Yunpeng Z, et al. Identification of IncRNA-associated competing triplets reveals global patterns and prognostic markers for cancer. Nucleic Acids Res. 2015;43(7):3478. doi:10.1093/nar/gkv233

42. Li CF, Li YC, Wang Y, Sun LB. The effect of LncRNA H19/ miR-194-5p axis on the epithelial-mesenchymal transition of colorectal adenocarcinoma. Cell Physiol Biochem. 2018;50(1):196-213. doi: $10.1159 / 000493968$

43. Marampon F, Codenotti S, Megiorni F, et al. NRF2 orchestrates the redox regulation induced by radiation therapy, sustaining embryonal and alveolar rhabdomyosarcoma cells radioresistance. $J$ Cancer Res Clin Oncol. 2019;145(4):881-893. doi:10.1007/s00432-019-02851-0

44. Sun D, Mu Y, Piao H. MicroRNA-153-3p enhances cell radiosensitivity by targeting BCL2 in human glioma. Biol Res. 2018;51(1):56. doi:10.1186/s40659-018-0176-5

45. Wang F, Mao A, Tang J, et al. microRNA-16-5p enhances radiosensitivity through modulating Cyclin D1/E1-pRb-E2F1 pathway in prostate cancer cells. J Cell Physiol. 2018.

46. Perez-Anorve IX, Gonzalez-De la Rosa CH, Soto-Reyes E, et al. New insights into radioresistance in breast cancer identify a dual function of miR-122 as a tumor suppressor and oncomiR. Mol Oncol. 2019;234(8):13182-13190.
OncoTargets and Therapy

\section{Publish your work in this journal}

OncoTargets and Therapy is an international, peer-reviewed, open access journal focusing on the pathological basis of all cancers, potential targets for therapy and treatment protocols employed to improve the management of cancer patients. The journal also focuses on the impact of management programs and new therapeutic

\section{Dovepress}

agents and protocols on patient perspectives such as quality of life adherence and satisfaction. The manuscript management system is completely online and includes a very quick and fair peer-review system, which is all easy to use. Visit http://www.dovepress.com/ testimonials.php to read real quotes from published authors. 